Evandro da S. F. Coutinho 1 Sidney Dutra da Silva 2 Carlos Henrique Assunção Paiva 3 Gustavo Gouvea de Freitas 3 Luciano Pereira Miranda 3 Rosania Mendonça 2

\section{Confiabilidade da informação sobre uso recente de medicamentos em um estudo caso-controle de base hospitalar}

\author{
Reliability of the information on the recent \\ use of medication in a hospital based \\ case-control study
}

1 Departamento de Epidemiologia e Métodos Quantitativos em Saúde, Escola Nacional de Saúde Pública, Fundação Oswaldo Cruz. Rua Leopoldo Bulhões, 1480 80 andar, Manguinhos,

Rio de Janeiro, R 21041-210, Brasil. 2 Universidade Aberta da Terceira Idade, Universidade do Estado do Rio de Janeiro. Rua São Francisco Xavier 524, 10 o andar, bloco F, Rio de Janeiro, RJ 20550-013, Brasil

3 Instituto de Medicina Social, Universidade do Estado do Rio de Janeiro. Rua São Francisco Xavier 524, 7 o andar, Rio de Janeiro, RJ, 20550-013, Brasil.

\begin{abstract}
Accuracy of the information is essential to produce unbiased estimates of the association between exposure and outcome. We are carrying out a case-control study which aim is to investi gate the association between the use of medication and falling injuries leading to hospitalisation in the elderly. As there is no gold-standard available, we estimated the reliability of the information on the use of these drugs within the 24 hours and two weeks before the fall using a test-retest strategy. Sixty-one individuals aged 60 years or more were re-intervi ewed within an interval of 5-7 days after the first interview. Kappa coefficients were high, showing a good consistence of collected data on medication recently used. Among the variables investi gated, only gender showed an association with reliability of the information, which was more consistent among women compared to men.
\end{abstract}

Key words Aged; Drugs; Domestic Accidents; Case-Control Studies

Resumo A qualidade da informação é fundamental para que se possa obter estimativas sem vieses da associação entre variáveis de exposi ção e de desfecho. Um estudo caso-controle está em curso com o objetivo de investigar uma possível associação entre o consumo de medicamentos e a hospitalização de idosos por fraturas decorrentes de quedas. Na ausência de um padrão-ouro, aval iou-se a confiabilidade da informação sobre o consumo desses medicamentos nas duas semanas que antecederam a queda, incluindo-se as úl timas 24 horas, por meio de uma estratégia teste- reteste. Reentrevistaram-se 61 idosos, com idade igual ou superior a sessenta anos, a um interval o de cinco a sete dias após a primeira entrevista. Os valores do coeficiente Kappa foram altos, mostrando uma el evada consistência da informação obtida sobre medi camentos usados recentemente. Dentre as variáveis investigadas, apenas gênero mostrou-se associada com a confiabilidade, sendo a informação mais consistente entre as mulheres do que entre os homens. Palavras-chave Idoso; Medicamentos; Acidentes Domésticos; Estudos de Caso e Controle 
Introdução

Aproximadamente um terço da população acima de 65 anos sofre pelo menos uma queda num período de um ano (Cumming et al., 1991; Tinetti, 1994; Tinetti et al., 1988; Northridge et al., 1995; Rozenfeld, 1997). As quedas costumam produzir uma redução na independência dos idosos, seja pelo receio que geram de novos acidentes, seja pelas lesões incapacitantes ou de difícil recuperação que podem causar (Tinetti, 1994).

Lesões graves decorrentes de quedas costumam ocorrer em cerca de 3-5\% dos idosos. Tais lesões incluem fratura de quadril, crânio, outras fraturas, luxações, lacerações (Tinetti, 1994; Tinetti et al., 1988; Sattin et al., 1990; Ryynänen et al., 1991). Sua freqüência, morbidade e elevado custo social e econômico fazem das quedas um importante problema de saúde pública nesse grupo etário. Estudos realizados em países escandinavos, EUA e Reino Unido mostram um crescimento anual de 1-4\% na incidência de fratura de bacia em homens, mesmo padronizando-se por idade. No caso de mulheres, os achados não são consistentes (Cumming et al., 1997).

Dentre os fatores que vêm sendo responsabilizados pelo aumento do risco de quedas e fraturas na população de idosos encontra-se o uso de medicamentos que provocam sonolência, alteram o equilíbrio, a tonicidade muscular e/ ou provocam hipotensão (Cumming et al., 1997).

Um estudo caso-controle está em andamento, com intuito de investigar o papel de um conjunto de medicamentos na ocorrência de fraturas que demandam internação de idosos. Para isso, a informação sobre os medicamentos consumidos nas 24 horas e nas duas semanas que antecederam a queda é vital.

Uma estratégia adotada por Rozenfeld (1997), em seu estudo, foi solicitar antecipadamente aos idosos que, ao comparecerem para a entrevista, trouxessem as receitas e caixas dos medicamentos que estavam usando. Esse procedimento foi facilitado pelo fato da pesquisa ter sido realizada num contexto mais favorável. A população do estudo era formada por mulheres idosas que freqüentavam a Universidade Aberta da Terceira Idade (UNATIUERJ), e que haviam sofrido, ou não, quedas durante o último ano.

Diferente do estudo de Rozenfeld, nosso estudo tem como população de referência os idosos hospitalizados por fraturas decorrentes de quedas (casos) ou por um conjunto de outras patologias pré-definidas (controles). O fato de estarem acamados num hospital torna mais difícil a verificação da veracidade da informação, pois não há como solicitar que os entrevistados tragam receitas ou caixas de remédios. Esta solicitação também não foi feita aos parentes, pois nem sempre estes eram encontrados durante o período das entrevistas. Por outro lado, visitas domiciliares após a alta teriam um custo elevado, havendo ainda uma probabilidade maior de esquecimento por parte desses idosos devido ao tempo transcorrido entre a hospitalização e a entrevista.

Em função dos problemas assinalados, optou-se por investigar a concordância da informação sobre o consumo de medicamentos nas 24 horas e nas duas semanas que antecederam a hospitalização dos casos e controles. Neste artigo, apresentaremos os resultados da confiabilidade da informação sobre os medicamentos usados em grupo de idosos no período que antecedeu à internação.

\section{Material e métodos}

Sessenta e um indivíduos, com 60 anos ou mais, internados em cinco hospitais públicos no município do Rio de Janeiro foram re-entrevistados, ainda no hospital, com intervalo de cinco a sete dias após a primeira entrevista. Desses, 32 foram selecionados como casos do estudo supracitado e 29 como controles. Os controles eram, em sua maioria, idosos internados para cirurgias de próstata, vesícula, hérnia, catarata, útero, ovário, períneo. Não houve recusas.

Nas duas ocasiões, adotou-se o mesmo procedimento para a coleta da informação sobre o consumo de medicamentos. Inicialmente, perguntava-se quais os remédios em uso nas 24 horas e nas duas semanas (excluídas as últimas 24 horas) anteriores à queda ou à hospitalização. Em seguida, o entrevistador repetia a pergunta de modo induzido para um conjunto de drogas de maior interesse para a pesquisa, por alterarem o equilíbrio, a tonicidade muscular, provocarem hipotensão ou sonolência.

A confiabilidade de "teste-reteste" foi medida através do coeficiente Kappa (Fleiss, 1981), calculado utilizando-se o "Computer Programs for Epidemiologic Analysis- Pepi" (Gahlinger \& Abramson, 1995). Os intervalos de confiança de $95 \%$ foram estimados através da abordagem baseada no teste 2 para adequação do ajuste ("chi-square goodness-of-fit test"), proposta por Donner \& Eliasziw (1992). Segundo os autores, esse método estima os intervalos de confiança em pequenas amostras com mais precisão do que os métodos propostos anteriormente, além 
de não requerer o pressuposto de uma distribuição aproximadamente normal do coeficiente Kappa. Comparou-se ainda a proporção de discordâncias segundo o tipo de paciente, o sexo, a escolaridade e a situação conjugal. Para essa análise, as discordâncias foram consideradas em conjunto, não havendo distinção entre aquelas referentes ao período de 24 horas ou de 2 semanas.

\section{Resultados}

Na Tabela 1, apresentamos as estimativas de Kappa para classes de medicamentos usados nas 24 horas que antecederam a internação. Houve um total de sete discordâncias envolvendo analgésicos, antiinflamatórios, benzodiazepínicos, diuréticos e medicamentos que constituíam a categoria "outros". Essas discordâncias ocorreram em relação às informações obtidas de seis dos 61 idosos re-entrevistados. O Kappa foi elevado mesmo onde houve discordância, sendo as menores estimativas pontuais para os diuréticos $(0,88)$ e anal gésicos $(0,89)$.

Na Tabela 2, estão as estimativas de Kappa referentes ao consumo nas duas semanas que antecederam a hospitalização (excluídas as últimas 24 horas). Houve quatro discordâncias observadas na informação obtida de quatro idosos. As classes de medicamentos para as quais ocorreu discordância foram as mesmas da informação referente às 24 horas, com exceção dos antiinflamatórios. A menor estimativa pontual também foi para os diuréticos $(0,88)$.

Não foi possível fazer uma análise mais extensa, com o intuito de identificar as variáveis associadas com a confiabilidade teste-reteste, por terem sido observadas apenas sete discordâncias, mesmo juntando-se os dois períodos cobertos pela pesquisa. Na Tabela 3, apresentam-se as razões de proporções não ajustadas. Apenas no caso da variável gênero, o intervalo de confiança não incluiu o valor nulo. O grupo de homens idosos apresentou uma proporção de discordâncias cerca de cinco vezes maior do que no grupo de mulheres ( $p=0,05$ ).

Não se detectou diferença na média de idade entre os grupos de idosos com e sem discordância na informação (72,5 x 73,1 anos, respectivamente; $p=0,81$ ). O mesmo ocorreu com o escore do instrumento BOAS (Veras et al., 1990), usado para avaliar a presença de demência senil $(p=0,25)$.

Tabela 1

Concordância da informação sobre o consumo de medicamentos nas 24 horas que antecederam a internação, obtida em duas ocasiões diferentes.

\begin{tabular}{|c|c|c|c|c|}
\hline Medicamento & $\begin{array}{l}\text { Concordância } \\
\text { utiliza }\end{array}$ & $\begin{array}{l}\text { Concordância } \\
\text { não utiliza }\end{array}$ & Discordância & Kappa (IC 95\%) \\
\hline Analgésico & 18 & 40 & 3 & $0,89(0,69-0,96)$ \\
\hline Antiácido & 4 & 57 & 0 & $1,00(0,61-1,00)$ \\
\hline Antialérgico & 0 & 61 & 0 & indeterminado \\
\hline Antianginoso & 11 & 50 & 0 & $1,00(0,81-1,00)$ \\
\hline Antidepressivo & 2 & 59 & 0 & $1,00(0,41-1,00)$ \\
\hline Antigripal & 0 & 61 & 0 & indeterminado \\
\hline Anti-hipertensivo & 26 & 35 & 0 & $1,00(0,88-1,00)$ \\
\hline Antiinflamatório & 11 & 49 & 1 & $0,95(0,73-0,99)$ \\
\hline Benzodiazepínico & 10 & 50 & 1 & $0,94(0,71-0,99)$ \\
\hline Cálcio & 0 & 61 & 0 & indeterminado \\
\hline Digitálico & 1 & 60 & 0 & $1,00(0,23-1,00)$ \\
\hline Diurético & 4 & 56 & 1 & $0,88(0,48-0,98)$ \\
\hline Laxante & 2 & 59 & 0 & $1,00(0,41-1,00)$ \\
\hline Miorrelaxante & 11 & 50 & 0 & $1,00(0,81-1,00)$ \\
\hline Vitamina D & 1 & 60 & 0 & $1,00(0,23-1,00)$ \\
\hline Vaso dilatador periférico & 4 & 57 & 0 & $1,00(0,61-1,00)$ \\
\hline O utros & 24 & 36 & 1 & $0,97(0,82-0,99)$ \\
\hline
\end{tabular}


Tabela 2

Concordância da informação sobre o consumo de medicamentos nas duas semanas que antecederam a internação (excluídas as últimas 24 horas), obtida em duas ocasiões diferentes.

\begin{tabular}{lrccc}
\hline Medicamento & $\begin{array}{c}\text { Concordância } \\
\text { utiliza }\end{array}$ & $\begin{array}{l}\text { Concordância } \\
\text { não utiliza }\end{array}$ & Discordância & Kappa (IC 95\%) \\
\hline Analgésico & 17 & 43 & 1 & $0,96(0,79-0,99)$ \\
Antiácido & 5 & 56 & 0 & $1,00(0,66-1,00)$ \\
Antialérgico & 0 & 61 & 0 & indeterminado \\
Antianginoso & 10 & 51 & 0 & $1,00(0,80-1,00)$ \\
Antidepressivo & 2 & 59 & 0 & $1,00(0,41-1,00)$ \\
Antigripal & 0 & 61 & 0 & indeterminado \\
Anti-hipertensivo & 26 & 35 & 0 & $1,00(0,88-1,00)$ \\
Antiinflamatório & 9 & 52 & 0 & $1,00(0,78-1,00)$ \\
Benzodiazepínico & 9 & 51 & 0 & $0,94(0,69-0,99)$ \\
Cálcio & 0 & 61 & 1 & indeterminado \\
Digitálico & 2 & 59 & 0 & $1,00(0,41-1,00)$ \\
Diurético & 4 & 56 & 0 & $1,08(0,48-0.98)$ \\
Laxante & 3 & 58 & 0 & $1,00(0,80-1,00)$ \\
Miorrelaxante & 10 & 51 & 0 & $1,00(0,23-1,00)$ \\
Vitamina D & 1 & 60 & 1 & $1,00(0,66-1,00)$ \\
Vasodilatador periférico & 5 & 56 & $0,98(0,82-0,99)$ \\
Outros & 28 & 32 & & 1 \\
\hline
\end{tabular}

Tabela 3

Proporção de discordâncias na informação sobre o consumo de medicamentos segundo variáveis sócio-demográficas*.

\begin{tabular}{|c|c|c|c|c|}
\hline Variável & $\mathrm{n}$ & \% de discordância & $\begin{array}{l}\text { Razão de } \\
\text { proporções } \\
\text { (IC de } 95 \%)\end{array}$ & $p$-valor \\
\hline \multicolumn{5}{|l|}{ Tipo de paciente } \\
\hline casos & 32 & 15,6 & $4,53(0,56-36,54)$ & 0,20 \\
\hline controles & 29 & 3,4 & 1 & \\
\hline \multicolumn{5}{|l|}{ Sexo } \\
\hline masculino & 17 & 23,5 & $5,18(1,04-25,70)$ & 0,05 \\
\hline feminino & 44 & 4,5 & 1 & \\
\hline \multicolumn{5}{|l|}{ Escolaridade } \\
\hline até primário completo & 44 & 11,4 & $1,93(0,24-15,35)$ & 1,00 \\
\hline ginásio incompleto ou + & 17 & 5,9 & 1 & \\
\hline \multicolumn{5}{|l|}{ Situação conjugal } \\
\hline casado & 30 & 14,8 & $2,22(0,44-11,18)$ & 0,41 \\
\hline viúvo & 27 & 6,7 & 1 & \\
\hline
\end{tabular}

* os períodos de 24 horas e duas semanas foram agrupados nesta tabela. 


\section{Discussão}

Os erros de classificação (viés de informação) da variável de exposição de um estudo casocontrole produzem distorções na estimativa das odds-ratios (OR). Se esses erros ocorrem igualmente entre casos e controles, existe a tendência de subestimar o efeito da exposição. Se esses erros ocorrem de modo desigual, o viés da OR estimada pode se dar tanto no sentido da sub quanto da superestimação do efeito. Portanto, a minimização desses erros é essencial para se conhecer a verdadeira magnitude do efeito desses medicamentos no risco de hospitalização por fraturas decorrentes de quedas entre idosos.

Nesse artigo, pelas razões já apontadas anteriormente, não se avaliou a dimensão dos erros de classificação (validade), mas a concordância da informação obtida em duas ocasiões diferentes (confiabilidade teste-reteste). De modo geral, as estimativas pontuais do coeficiente Kappa mostraram uma confiabilidade elevada para as classes de medicamentos investigadas. Em alguns casos, os intervalos de confiança foram muito amplos, sobretudo em função do método de estimativa que escolhemos (goodness- of fit approach). Embora o método baseado nos erros-padrão, em muitos casos, fornecesse interval os de menor amplitude, estes podem ser menos precisos em amostras pequenas, e requerem que o Kappa tenha distribuição aproximadamente normal. Por isso, optamos por utilizar o método com estimativas mais conservadoras.

No caso dos analgésicos, dos antianginosos, dos antiinflamatórios e dos miorrelaxantes, houve um número um pouco maior de pa-

\section{Agradecimentos}

Os autores agradecem ao CNPq pelo apoio financeiro à pesquisa (processo oo 523024/ 96-7). cientes que referiram o seu uso nas últimas 24 horas, em comparação com o período de duas semanas (excluídas as últimas 24 horas). Isso pode indicar um viés de memória com maior chance de esquecimento para períodos mais distantes. Ainda que isso deva ser considerado quando se avalia a qualidade da informação, acreditamos que tal viés seja menos grave no caso do nosso estudo, pois o interesse maior recai sobre a possível associação entre o consumo nas últimas 24 horas.

Não foi possível explorar de modo mais aprofundado o impacto de variáveis sócio-demográficas, assim como a inserção no estudo (casos $x$ controles), na confiabilidade da informação sobre o uso de medicamentos. Como já assinalamos, o número de discordâncias foi muito pequeno. Dentre as variáveis exploradas, a única para a qual foi possível identificar esta associação foi o gênero, com uma consistência maior da informação entre as mulheres em relação aos homens. Esse achado é coerente com a literatura, pois vários estudos relatam que as mulheres são mais atentas e preocupadas com as questões envolvendo a saúde do que os homens.

Concluindo, a confiabilidade da informação sobre o uso de medicamentos nas 24 horas e nas duas últimas semanas foi elevada. Porém, é importante assinalar que uma confiabilidade elevada não significa que a informação seja válida, correta. Ela apenas indica o grau de consistência dessa informação. Entretanto, como a confiabilidade elevada é uma condição necessária, embora não suficiente, para que a informação seja considerada válida, esta deve sempre ser avaliada na impossibilidade de se conhecer a validade.

\section{Referências}

CUMMING, R. G.; MILLER, J. P.; KELSEY, J. L.; DAVIS, P.; ARFKEN, C. L.; BIRGE, S. J. \& PECK, W. A., 1991. Medications and multiple falls in elderly people: The St. Louis OASIS Study. Age and Aging, 20:455461.

CUMMING, R. G.; NEVITT, M. C. \& CUMMING, S. R., 1997. Epidemiology of hip fractures. Epidemiologic Reviews, 19:244-257.

DONNER, A. \& ELIASZIW, M., 1992. A goodness-of-fit approach to inference procedures for the Kappa statistic: Confidence interval construction, significance-testing and sample size estimation. Statistics in Medicine, 11:1511-1519. 
FLEISS, J. L., 1981. Statistical Methods for Rates and Proportions. New York: John Wiley and Sons.

GAHLINGER, P. M. \& ABRAMSON, J. H., 1995. Computer Programs for Epidemiologic Analysis - Pepi, version 2.05. Georgia: USD Inc.

NORTHRIDGE, M. E.; NEVITT, M. C.; KELSEY, J. L. \& LINK, B., 1995. Home hazards and falls in the elderly: The role of health and functional status. American Journal of Public Health, 85:509-515.

ROZENFELD, S., 1997. Reações Adversas aos Medicamentos na Tercei ra Idade: As Quedas Como Iatrogenia Farmacoterapêutica. Tese de Doutorado, Rio de Janeiro: Instituto de Medicina Social, Universidade do Estado do Rio de Janeiro.

RYYNÄNEN, O. P.; KIVELÄ, S. L.; HONKANEN, R.; LAIPPALA, P. \& SOINI, P., 1991. Incidence of falling injuries leading to medical treatment in the elderly. Public Health, 105:373-386.
SATTIN, R. W.; HUBER, D. A. L.; DEVITO, C. A.; RODRIGUEZ, J. G.; ROS, A.; BACCHELI, S.; STEVENS, J. A. \& WAXWEILER, R. J., 1990. The incidence of fall injury events among the elderly in a defined population. American Journal of Epidemiology, 131:1028-1037.

TINETTI, M. E., 1994. Prevention of falls and fall injuries in elderly persons: A research agenda. Preventive Medicine, 23:756-762.

TINETTI, M. E.; SPEECHLEY, M. \& GINTER, S. F., 1988. Risk factors for falls among elderly persons living in the community. New England Journal of Medicine, 319:1701-1707.

VERAS, R. P.; COUTINHO, E. S. F. \& NEY J r., G., 1990. População idosa no Rio de Janeiro (Brasil): Estudo piloto da confiabilidade e validação do segmento de saúde mental do questionário Boas. Revista de SaúdePública, 24:156-163. 